\title{
The Relationship Between Previous Injuries and Acromiohumeral Distancing in Adolescent Athletes
}

\section{Ahmed Abd-Elkhalek Mansour ${ }^{1}$, El-Saeed Tamer Mohamed ${ }^{2}$ and Abdelazeim Faten Hassan ${ }^{2 *}$}

${ }^{1}$ Physical Therapy Unit, Bab El-Sharie University Hospital, Al-Azhar University,

Egypt

${ }^{2}$ Department of Physical Therapy for Pediatrics, Faculty of Physical Therapy, Cairo

University, Egypt

*Corresponding Author: Abdelazeim Faten Hassan, Department of Physical

Therapy for Pediatrics, Faculty of Physical Therapy, Cairo University, Egypt.
Received: January 12, 2021

Published: February 20, 2021

(C) All rights are reserved by Abdelazeim

Faten Hassan., et al.

\begin{abstract}
Background: Following injury, there were strength, proprioception, and kinematics that lead to overall changes in function and motor control. The researchers in this study aimed to investigate the correlation between the previous knees, back and shoulder injuries to acromio-humeral distance measurement (AHD) in adolescent athletes.

Methods: Thirty-nine rowers were recruited during the national championship of indoor rowing with mean age $16.35 \pm 1.39$ years. AHD at $0^{\circ}$ and $90^{\circ}$ abduction from the scapular plane of both shoulders was measured pre-and post- rowing by ultrasound and a history of previous injuries was taken. The correlation was investigated between number of previous injuries from different categories and mean difference of AHD.

Results: At angle 0 abduction in either right or left shoulder, there was a weak association (Eta $=0.135)$ between previous injuries and AHD also the previous injuries had an effect of 1.8\% on AHD but at angle 90, the effect was higher in both left and right shoulder (6.8\% and $5.1 \%$ respectively).

Conclusion: Even with a weak correlation founded between previous injuries and AHD, a percent of effect was founded for previous injuries which may be clinically important.
\end{abstract}

Keywords: Acromio-humeral Distance; Previous Injury; Adolescent Athletes; Rowing

\section{Abbreviation}

AHD: Acromio-humeral Distance; SD: Standards; CM: Centimeter; MD: Mean Difference; RT: Right; LT: Left.

\section{Introduction}

Rowing ergometers simulate boat rowing movements on the land that are widely used at indoors national and international championships, most training centers. Besides, rowing needs high levels of training and commitment. Rowers have high-risk factors to injuries of the musculoskeletal system due to their high training volume and stresses, the most frequently lifetime prevalence of rower injuries was reported to be lower back (87\%), followed by the knee (65\%) and shoulder (61\%) [1]. Unless this high injury prevalence, there is less attention paid to injuries and their associated risk factors in adolescent athletes as finding the risk factors is a very important step in the prevention of injury [2]. 
Traditional rehabilitation programs focus only on a structure that involved or injured, however, the body moves in a dynamic integrated manner which referred to as kinetic chain, an efficient Kinetic chain will generate and allow efficient energy transfer throughout the whole chain related to function and the reverse will occur if inefficient chain found at any part of the body leads to more loads and injuries $[3,4]$.

Energy transfer through shoulder complex with high rate has been noted during the practice of motor activities in injured compared with their uninjured athletes [3]. In overhead athletes, upper limb injuries risk and pain increased in players with injuries of lower limb [3-8]. In rowers, the force generated by the lower limb and trunk is transferred to the oar through the shoulder joints [9].

Following-injury, there were strength, proprioception, and kinematics changes, which may have an effect and overall changes in motor control and function [10]. History of injury in Youth playing Soccer was associated with an increased rate of injuries, this suggests that those with a history of injuries may be at higher risk [11]. Also, there is a conclusion for injury risk factors is previous injuries in athletes [12-14].

Acromiohumeral distance (AHD) is referred to be the longitudinal line from the tip of the head of the humerus to the most lateral edge of the acromion measured by ultrasound which was the preferred technique and widely used in the measurement of this distance [15-17]. Maintaining AHD is a shoulder rehabilitation exercise goal and subacromial impingement prevention tool as individuals with subacromial impingement syndrome have been mentioned to have decreased AHD from $30^{\circ}$ to $90^{\circ}$ of active upper extremity elevation [18].

This study aimed to measure the correlation between the number of previous injuries concerning knee, back, and shoulder regions to AHD measurement in adolescent rowers which may help in identifying risk factors for injuries that will help in designing prevention programs.

\section{Materials and Methods}

\section{Study participants and recruitment criteria}

Thirty nine adolescent rowers of both sexes aged from $14-18$ years old were selected from indoor national championship to ac- complish the study. Volunteers were included if they met age criteria, had no pain at the time of examination. Also, they were excluded if they had previous shoulder complex, cervical and thoracic fractures, or surgeries, either cervical pain or arm radiculopathy, history of shoulder dislocation, and a sign of fatigue pre-examination. All participants rowed against their competitors for 1000 meters at the ergometer indoor championship to ensure maximum workout and so more loads and stresses. A written consent form was obtained from all participants and their coaches.

\section{History taking}

Pre-the race; History of the shoulder, knee, and back injuries last two seasons was taken from the athletes by asking the volunteers which part was injured, how many, and since what? The injury was defined as a problem that caused the athlete to miss at least one competition or at least two training sessions or required at least one visit to a health professional for treatment. This definition was adapted from the Rugby Injury and Performance Project [19].

\section{Assessment tools}

Musculoskeletal ultrasound device (Mindray DP10, with a linear probe, at frequency $10 \mathrm{MHZ}$ (serial number: bn-75013216), China) was used to measure AHD pre- and post- rowing race for both shoulders at both $0^{\circ}$ and $90^{\circ}$ horizontal abduction $\left(40^{\circ}\right.$ front of the frontal plane) with thumb up and elbow extended from sitting in an erect position with the foot planted in the ground [20], and the examiner was blinded by covering the site of measurement on the ultrasound screen by adhesive tape (Figure 1). The examiner had 3 years of experience in sonography and trained for approximately 2 months for the current study's purpose before this data collection. Shoulder position angles were measured by universal goniometer and all volunteers were instructed, motivated, and observed to actively maintain the shoulder position angle during ultrasound measurement.

\section{Statistical design}

Data were collected and statistically analyzed. Descriptive statistics in form of means, standard deviation (SD), and frequencies were carried to summarize the data.

Shapiro-Wilk's test ( $p>0.05$ ) was used to assess the distribution of AHD. Levene's ( $p>0.05$ ) was used to assess the homogeneity of variances. Scatterplot was used to assess the relationship between the dependent variables. 
36
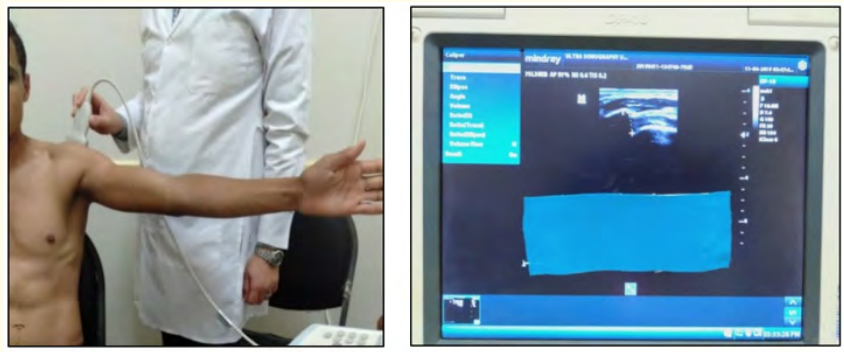

Figure 1: Ultrasound measurement at $90^{\circ}$ shoulder scapular position using adhesive tape to the blind examiner.

An evidence of multicollinearity was assessed by Pearson correlation $(|\mathrm{r}|<0.9)$, univariate or multivariate outliers was assessed by inspection of a boxplot and Mahalanobis distance and there were no univariate or multivariate outliers in the data. Eta was used to calculate correlation between number of previous injuries from different origins and mean difference of AHD (pre- and post- race).

\section{Results}

Thirty-nine participants (volunteers) were conducted in this study (33 males and 6 females) with mean age, body mass, height, and years of experience values of $16.35 \pm 1.39$ years, $70.87 \pm 12.28$ $\mathrm{kg}, 170.21 \pm 10.52 \mathrm{~cm}$, and $3.67 \pm 0.94$ years respectively.

Descriptive data for AHD measurement at $0^{\circ}$ and $90^{\circ}$ at different measuring periods (pre and post rowing) at both sides are shown in table 1.

The distribution of AHD was normally distributed at $0^{\circ}$ and $90^{\circ}$. Besides, there was homogeneity of variances. There was a linear relationship between the dependent variables.

Eta value is 0.135 between previous injuries and mean difference (MD) of AHD at the left shoulder, it is inferred that there is a weak association, then after taking the square of Eta of 0.135 , the result is 0.018 , which means that the independent variable (previous injuries) has $1.8 \%$ effect on the dependent variable (MD of AHD) of left shoulder at $0^{\circ}$ and it is the same for right shoulder at $0^{\circ}$.

\begin{tabular}{|c|c|c|c|c|}
\hline \multirow{2}{*}{ Angle } & \multirow{2}{*}{$\begin{array}{c}\text { Measuring } \\
\text { Side }\end{array}$} & \multicolumn{2}{|c|}{ (Mean \pm SD) } & \multirow{2}{*}{$\begin{array}{c}\text { Mean Differ- } \\
\text { ence (MD) }\end{array}$} \\
\cline { 3 - 5 } & & Pre & Post & 0.44 \\
\hline \multirow{2}{*}{$0^{\circ}$} & Left side & $10.89 \pm 0.57$ & $11.33 \pm 0.51$ & 0.50 \\
\cline { 2 - 5 } & Right side & $10.57 \pm 0.38$ & $11.07 \pm 0.41$ & 1.01 \\
\hline \multirow{2}{*}{$90^{\circ}$} & Left side & $10.23 \pm 0.46$ & $11.24 \pm 0.32$ & 0.42 \\
\cline { 2 - 5 } & Right side & $10.31 \pm 0.33$ & $10.73 \pm 0.46$ & \multicolumn{2}{|c}{} \\
\hline
\end{tabular}

Table 1: Descriptive statistics for AHD at $0^{\circ}$ and $90^{\circ}$ at different measuring periods.

Left shoulder at $90^{\circ}$ Eta $=0.262$ and the square 0.068 which means that its effect is $6.8 \%$ but at right shoulder $90^{\circ} \mathrm{Eta}=0.227$ and the square $=0.051$ so its effect $=5.1 \%$.

\section{Discussion}

In this study, the authors investigated the correlation between previous injuries involving the shoulder, knees, and back and the mean difference of pre-and post-rowing competition results of acromio-humeral distance to study the clinical impact of previous injuries on the performance of all participants also the result may help in identifying risk factors for injuries that will help in designing prevention programs.

Hägglund., et al. said that a previous injury is an important risk factor for injuries in football players but up to the available knowledge no previous studies discussed the risk of previous injuries to shoulder injuries in rower athletes and to discuss this effect the previous injuries association and effect to AHD were assessed at the present study [21].

In current clinical practice, a majority of the preventive rehabilitation interventions commonly utilized the previous injuries as a point to focus as recommended by DiFiori., et al. who said, the history of prior injury is an established risk factor for overuse injuries also at elite athletes [22].

According to the results of the current study, at angle 0 abduction in RT or LT shoulder, there was a weak association (Eta 
$=0.135$ ) between previous injuries and AHD and the previous injuries had an effect of $1.8 \%$ on AHD but at an angle 90 , the effect was higher in both LT\&RT shoulder (6.8\% and 5.1\% respectively), which means that the effect increased by the increasing of abduction angle and to illustrate this results we hypothesized that with increasing abduction angle the muscles act actively and the role of a kinetic chain be causing more loads transition to the muscles that control AHD as there's muscle balance alteration due to previous injuries which it is supported by the Fulton., et al. findings [10].

Unless the effect of previous injuries on AHD of the adolescent rower is small it may be one factor of multiple risk factors for future shoulder injuries and may be clinically important if other risk factors founded in the same athlete leading to future shoulder injuries as injuries in athletes is multimodal as said by DiFiori., et al. [22].

A proximal segments motion breakdown (like core, legs, and spine) that happened post injuries leads to increasing the stresses placed on the distal segments of the body (like shoulder, elbow, and wrist) and this may illustrate the effect of previous injuries in AHD that founded in the current study results $[23,24]$.

Poor endurance in core muscles is likely associated with nonspecific back disorders in collegiate athletes [25]. Also, greater shoulder dysfunction is correlated with back core stabilizers weakness [26]. This comes in agreement with our study's result that previous injuries like back injuries have an association and effect in shoulder AHD.

The most important muscles at the beginning of the rowing race were the leg and shoulder muscles, In the middle of the race the knee and elbow flexors were the most active muscles to maintain the pace constant, but at the finishing part or the last minute, the leg muscles and the extensors of the shoulder were involved again so any alteration at this sequenced patterns during the race that may result from previous injuries may lead to more loads and more reliance on the shoulder joint as it's one of the most involved joints at the start or the finish of the race and the authors hypothesized that may be one of the theories that demonstrate the effect of previous injuries in rower's AHD appeared as a result of this current study [27].
The biomechanical interrelationships between body regions and the kinetic link model which depicts that efficient motion and efficient muscle activation are believed to occur in the proximal to distal sequence pattern in normal athletes also voluntary upper extremity movements in the normal motor pattern come after muscle activation of lower limb and trunk, so any changes in this motor programing or sequence after injuries may lead to changes in AHD as mentioned in this research [28].

Into consideration, this study had some limitations, the sample was small and categorization of injuries is not concerned in our study as the effect of certain body part injuries is lacked.

\section{Conclusion}

We concluded that, a weak association founded between previous injuries involving the shoulder, knees, and back and AHD of adolescent's rower. In addition, a percent of effect was founded for these previous injuries on AHD which may be clinically important. The authors recommend that, coaches and the medical teams of young rower athletes should focus on athletes that previously injured and properly decided when to return to practice. More studies in future with large sample size and different evaluative tools are recommended to confirm obtained results. Also, the criterion for different injuries is recommended.

\section{Acknowledgment}

We appreciate all efforts of the volunteers and the co-operation of their coaches to accomplish this work.

\section{Conflict of Interest}

There are no conflicts of interest.

\section{Bibliography}

1. Trompeter K., et al. "Prevalence of musculoskeletal pain in elite rowers". British Journal of Sports Medicine 51 (2017): 398-398.

2. Van Mechelen W., et al. "Incidence, severity, etiology and prevention of sports injuries. A review of concepts". Sports Medicine (Auckland, N.Z.) 14.2 (1992): 82-99. 
3. Chu Samuel K., et al. "The Kinetic Chain Revisited: New Concepts on Throwing Mechanics and Injury". PM and R: The Journal of Injury, Function, and Rehabilitation 8.3 (2016): S69-77.

4. Martin Caroline., et al. "Energy flow analysis during the tennis serve: comparison between injured and noninjured tennis players". The American Journal of Sports Medicine 42.11 (2014): 2751-2760.

5. Andersson Stig Haugsboe., et al. "Preventing overuse shoulder injuries among throwing athletes: a cluster-randomised controlled trial in 660 elite handball players". British Journal of Sports Medicine 51.14 (2017): 1073-1080.

6. Endo Yasuhiro and Masaaki Sakamoto. "Correlation of shoulder and elbow injuries with muscle tightness, core stability, and balance by longitudinal measurements in junior high school baseball players". Journal of Physical Therapy Science 26.5 (2014): 689-693.

7. Laudner Kevin., et al. "The relationship between clinically measured hip rotational motion and shoulder biomechanics during the pitching motion". Journal of Science and Medicine in Sport 18.5 (2015): 581-584.

8. Lintner Dave., et al. "Injury patterns and biomechanics of the athlete's shoulder". Clinics in Sports Medicine 27.4 (2008): 527-551.

9. Hosea Timothy M and Jo A Hannafin. "Rowing injuries". Sports Health 4.3 (2012): 236-45.

10. Fulton Jessica., et al. "Injury risk is altered by previous injury: a systematic review of the literature and presentation of causative neuromuscular factors". International Journal of Sports Physical Therapy 9.5 (2014): 583-595.

11. Kucera K L., et al. "Injury history as a risk factor for incident injury in youth soccer". British Journal of Sports Medicine 39.7 (2005): 462.

12. Hjelm N., et al. "Injury risk factors in junior tennis players: a prospective 2-year study". Scandinavian Journal of Medicine and Science In Sports 22.1 (2012): 40-8.
13. Jacobsson Jenny., et al. "Injury patterns in Swedish elite athletics: annual incidence, injury types and risk factors". British Journal of Sports Medicine 47.15 (2013): 941-952.

14. Von Rosen Philip and Annette Heijne. "Previous and current injury and not training and competition factors were associated with future injury prevalence across a season in adolescent elite athletes". Physiotherapy Theory and Practice 1-8. 18 May. (2020).

15. McCreesh Karen M., et al. "Acromiohumeral distance measurement in rotator cuff tendinopathy: is there a reliable, clinically applicable method? A systematic review". British Journal of Sports Medicine 49.5 (2015): 298-305.

16. Navarro-Ledesma S., et al. "Is coracohumeral distance associated with pain-function, and shoulder range of movement, in chronic anterior shoulder pain?". BMC Musculoskeletal Disorders 18.1 (2017).

17. Hébert Luc J., et al. "Acromiohumeral distance in a seated position in persons with impingement syndrome". Journal of Magnetic Resonance Imaging: JMRI 18.1 (2003): 72-79.

18. Michener Lori A., et al. "Anatomical and biomechanical mechanisms of subacromial impingement syndrome". Clinical Biomechanics (Bristol, Avon) 18.5 (2003): 369-379.

19. Wilson F., et al. "A 12-month prospective cohort study of injury in international rowers". British Journal of Sports Medicine 44.3 (2010): 207-214.

20. Alibazi Razie J., et al. "The Effect of Shoulder Muscle Fatigue on Acromiohumeral Distance and Scapular Dyskinesis in Women With Generalized Joint Hypermobility". Journal of Applied Biomechanics 33.6 (2017): 424-430.

21. Hägglund M., et al. "Previous injury as a risk factor for injury in elite football: a prospective study over two consecutive seasons". British Journal of Sports Medicine 40.9 (2006): 767-772.

22. DiFiori John P., et al. "Overuse injuries and burnout in youth sports: a position statement from the American Medical Society for Sports Medicine". British Journal of Sports Medicine 48.4 (2014): 287-288. 
23. Van der Hoeven $\mathrm{H}$ and $\mathrm{W}$ B Kibler. "Shoulder injuries in tennis players”. British Journal of Sports Medicine 40.5 (2006): 43540; discussion 440 .

24. Kibler W Ben., et al. "Mechanics and pathomechanics in the overhead athlete". Clinics in Sports Medicine 32.4 (2013): $637-$ 651.

25. Abdelraouf Osama Ragaa and Amr Almaz Abdel-Aziem. "The relationship between core endurance and back dysfunction in collegiate male athletes with and without nonspecific low back pain". International Journal of Sports Physical Therapy 11.3 (2016): 337-344.

26. Radwan Ahmed., et al. "Is there a relation between shoulder dysfunction and core instability?". International Journal of Sports Physical Therapy 9.1 (2014): 8-13.

27. Gerževič Mitja., et al. "Differences in muscle activation between submaximal and maximal 6-minute rowing tests". Journal of Strength and Conditioning Research 25.9 (2011): 2470-2481.

28. McMullen J and T L Uhl. "A kinetic chain approach for shoulder rehabilitation". Journal of Athletic Training 35.3 (2000): 329-

337.

\section{Assets from publication with us}

- Prompt Acknowledgement after receiving the article

- Thorough Double blinded peer review

- Rapid Publication

- Issue of Publication Certificate

- High visibility of your Published work

Website: www.actascientific.com/

Submit Article: www.actascientific.com/submission.php

Email us: editor@actascientific.com

Contact us: +919182824667 